\title{
Androstenedione Measurement
}

National Cancer Institute

\section{Source}

National Cancer Institute. Androstenedione Measurement. NCI Thesaurus. Code C74843.

The determination of the amount of androstenedione hormone present in a sample. 